\title{
Helium flux and elemental composition of galactic Cosmic Rays with the DAMPE space mission
}

\author{
Margherita Di Santo ${ }^{1,2, a}$, on behalf of the DAMPE collaboration \\ ${ }^{1}$ Dipartimento di Matematica e Fisica "E.De Giorgi", Università del Salento, 73100, Lecce, Italy \\ ${ }^{2}$ Istituto Nazionale di Fisica Nucleare (INFN) - Sezione di Lecce, I-73100, Lecce, Italy
}

\begin{abstract}
DAMPE (DArk Matter Particle Explorer) is a space mission project promoted by the Chinese Academy of Sciences (CAS), in collaboration with Universities and Institutes from China, Italy and Switzerland. The detector is collecting data in a stable sun-synchronous orbit lasting 95 minutes at an altitude of about $500 \mathrm{~km}$. It has been launched in December 17th, 2015, from the Jiuquan Satellite Launch Center, in the Gobi Desert. The main goals of the mission are: indirect search for Dark Matter, looking for signatures in the electron and photon spectra with energies up to $10 \mathrm{TeV}$; analysis of the flux and composition of primary Cosmic Rays with energies up to hundreds of $\mathrm{TeV}$; high energy gamma-ray astronomy. Preliminary results about the Helium flux and Cosmic Ray composition will be presented and discussed.
\end{abstract}

\section{Introduction}

Helium nuclei (He), together with Protons (p), are the most abundant components of Cosmic Rays (CRs). The study of their fluxes as a function of their kinetic energy is crucial to better understand where these particles come from and which are the accelaration and propagation mechanisms that rule their path all around the Galaxy. Several experiments (like AMS [1], CREAM [2], PAMELA [3] and ATIC [4]) found that the measured spectral shape of cosmic He nuclei cannot be well described by a single power law because data exhibit a spectral hardening at energies of hundreds of GeV. This feature of He flux (seen also in the p flux) gives rise to different and complex models which try to clarify the origin and the acceleration and diffusion processes of CRs. Here the DAMPE preliminary results about the elemental composition of CRs and the He flux will be presented and discussed.

\section{The DAMPE detector}

The DArk Matter Particle Explorer (DAMPE) [5] spacecraft hosts a powerful space telescope consisting of four sub-detectors, as schematically shown in Fig. 1, which are, from the top to the bottom of the satellite, the following:

1) Plastic Scintillation array Detector (PSD), composed by 82 bars of plastic scintillator assembled on two planes with a double layer configuration. It is used to measure the absolute value of the

\footnotetext{
ae-mail: margherita.disanto@le.infn.it
} 


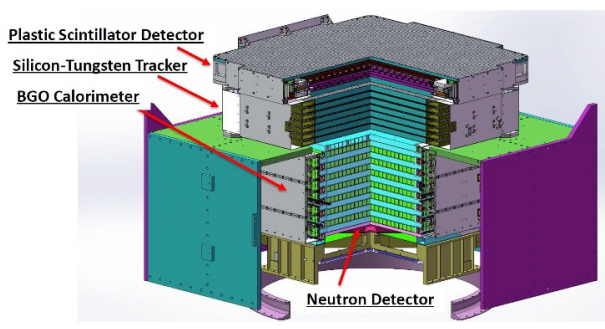

Figure 1. 3D view of the DAMPE detector.

charge of incident high-energy particles (with $Z \leq 28$ ) with a charge resolution $\leq 25 \%$ for $Z=1$, as well as to provide an anti-coincidence system for $\gamma$-rays.

2) Silicon-Tungsten tracKer-converter (STK), composed by twelve position-sensitive silicon detector planes and three thin layers of tungsten inserted in between the silicon planes (2, 3, 4 and 5) to increase the convertion probability of $\gamma$-rays in $e^{+} / e^{-}$pairs. The main purpose of the STK is the trajectory reconstruction of incoming particles and a further measurement of their charge.

3) $B G O$ calorimeter $(B G O)$, made up of 14 layers with a total area of $60 \mathrm{~cm} \times 60 \mathrm{~cm}$ and a depth of $\sim 32$ radiation lengths and $\sim 1.6$ interaction lengths $(\sim 40 \mathrm{~cm})$. Each layer constists of 22 crystal bars of Bismuth germanium oxide $\left(\mathrm{Bi}_{3} \mathrm{Ge}_{4} \mathrm{O}_{12}, \mathrm{BGO}\right)$. It provides the energy measurement, the electron and hadron identification and an additional contribution to the track reconstruction. It guarantees very good performances in the energy ranges of $1 \mathrm{GeV}-10 \mathrm{TeV}$ for electrons and $\gamma$ and $50 \mathrm{GeV}-100 \mathrm{TeV}$ for nuclei.

4) NeUtron Detector (NUD) made of boron-doped plastic scintillator plates. It gives further information for electron/hadron discrimination.

\section{DAMPE data taking and event selection criteria}

The DAMPE satellite provides a good geometrical acceptance of $\sim 0.3 \mathrm{~m}^{2} \mathrm{sr}$ for galactic CRs events in the energy range $20 \mathrm{GeV}-100 \mathrm{TeV}$. It is stably collecting $\sim 5 \mathrm{M}$ of events every day with a total amount of $\sim 100 \mathrm{~GB} /$ day of data transferred to the ground, with a trigger rate of $\sim 50 \mathrm{~Hz}$. The dead time of the satellite is mainly due to three contributions: the lapse in which the detector crosses the South Atlantic Anomaly (SAA) region ( 4.5\% of the operation time), the on-orbit calibration datataking $(\sim 1.5 \%)$ and the instrumental dead time $(\sim 18.5 \%)$.

In this work, every event recorded by DAMPE inside the SAA region is excluded. This is one of the so-called pre-selection cuts, together with the rejection of side track events and the requirement of total energy depositon $\mathrm{E}_{d e p}>20 \mathrm{GeV}$ inside the $\mathrm{BGO}$ calorimeter. Once an event satisfies all the preselection requirements, the subsequent analysis goes through several steps made up of the following requests :

- High Energy Trigger (HET), which means an energy release higher than $\sim 10 \mathrm{MIP}\left(1 \mathrm{MIP}_{\mathrm{BGO}}=\right.$ $23 \mathrm{MeV}$ ) inside the first 4 layers of the BGO;

- at least one reconstructed track in the STK;

- compatibility between the STK track direction and the position of the hit on the PSD bars; 
- match between the direction of the STK track and the direction of the BGO shower and, in case of more than one track, the closest to the BGO shower direction will be selected;

- match of the PSD charge measurement with the first STK layer charge measurement;

- discrimination between electromagnetic and hadronic induced event;

- choice of a proper PSD energy range to select the charge and so the incoming particles of interest.

The use of these event selection criteria is supported by the good agreement between the results of the analysis of flight-data and Monte-Carlo simulations.

\section{Preliminary Charge Spectrum and Helium flux with DAMPE}

A good estimate of the charge spectrum up to $Z=28$ (Ni) has been obtained by using the charge measurement provided by the PSD. The top-most sub-detector of DAMPE is composed by 2 planes, each one with 41 bars of plastic scintillator. The bars on the top plane are parallel to the X-axis of the satellite coordinate system, while that of the second plane are parallel to the Y-axis. For this reason, the two planes can be called the $\mathrm{YZ}$ and the $\mathrm{XZ}$ views, respectively. The best estimate of the incoming particle charge can be provided by the arithmetic mean of the energy loss measurements in the two views XZ and YZ of the PSD. A preliminary result of the charge spectrum, shown in Fig. 2, has been obtained as the number of events recorded by DAMPE during 17 months as a function of the square root of the arithmetic mean of the two PSD views divided by $1 \mathrm{MeV}$ in order to have a dimensionless number which is, according to the Bethe-Bloch formula, proportional to the charge of the incoming CR nucleus. Almost all the peaks are clearly visible up to Nickel.

Preliminary results about He acceptance and flux are shown in Fig. 3. The effettive acceptance, Fig. $3 a$, has been estimated by using a Monte-Carlo He sample and the following formula:

$$
A_{E F F}=A_{G E N} \frac{N_{S U R V}}{N_{G E N}}
$$

where $A_{G E N}$ is the Acceptance for the generated MC events, $N_{G E N}$ is the number of the generated events and $N_{S U R V}$ is the number of all the generated events which survived to the same analysis cuts used for the on-orbit data.

The He flux measured by DAMPE as a function of the kinetic energy per nucleon, Fig. $3 b$, with a systematic uncertainty of $\sim 15 \%$, shows a good agreement with other experiments. The statistical errors are plotted with bars while the systematics are represented by the grey band. Systematic errors have been evaluated by considering the differences between on-orbit data and MC data, taking into account the uncertainty due to the HET, the STK track selection and the PSD charge reconstruction in its two layers. Also the proton contamination in the Helium sample has been evaluated by using a mixed proton and Helium MC sample. The result is that this contamination is very small $(<1.5 \%)$, while the Lithium pollution at low energies results negligible. A spectral hardening after $\sim 100 \mathrm{GeV} / \mathrm{n}$ can be observed, confirming the previous results.

\section{Conclusions}

The DAMPE satellite is in a very stable data-taking since December 2015, providing satisfactory preliminary results about the All-Z charge spectrum and the He analysis, which have been presented 


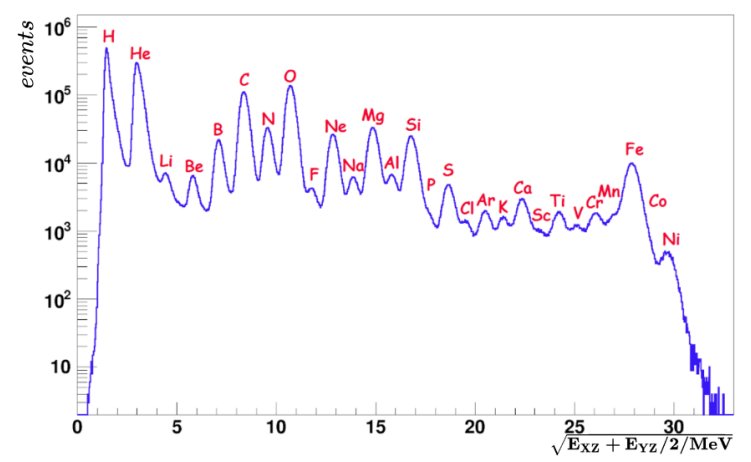

Figure 2. The All-Z charge spectrum reconstructed with the first 17 months of on-orbit data by using the arithmetic mean of the energy deposition measured in the $\mathrm{XZ}\left(\mathrm{E}_{\mathrm{XZ}}\right)$ and $\mathrm{YZ}\left(\mathrm{E}_{\mathrm{YZ}}\right)$ views of the PSD.

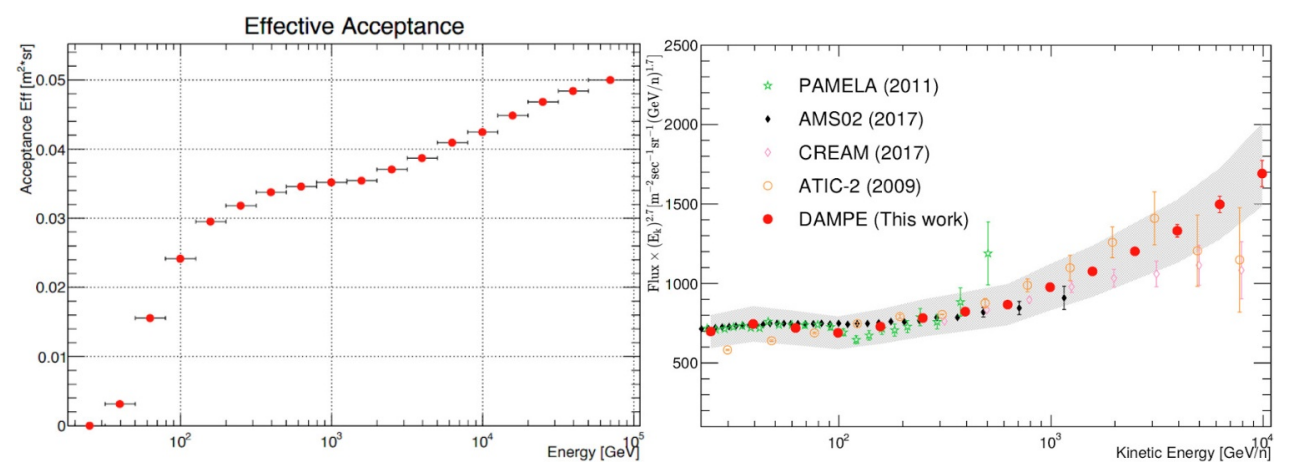

Figure 3. (a) Preliminary Cosmic Ray Helium effective acceptance. (b) Preliminary He flux as a function of the kinetic energy per nucleon measured by DAMPE compared with other experiments. The statistical errors are plotted with bars while the systematics are represented by the grey band.

here. By taking into account the charge measurement provided by the PSD, a very good charge spectrum has been obtained, with the peaks of almost all the nuclei from $\mathrm{H}$ to $\mathrm{Ni}(Z=1-28)$ clearly visible. The preliminary He flux shows a good agreement with the previous experiments, confirming the hardening observed after $\sim 100 \mathrm{GeV} / \mathrm{n}$. One of the next goals of the DAMPE mission is to go further with energies up to $100 \mathrm{TeV} / \mathrm{n}$ in order to provide an important contribution to the knowledge about the origin, the accelaration and propagation mechanisms of Cosmic Rays, as well as about their elemental composition.

\section{References}

[1] M. Anguilar et al. (AMS Collaboration), Phys. Rev. Lett. 115 (2015) 211101

[2] Yoon et al. (CREAM Collaboration), Astrophys.J. 839 (2017) 1

[3] O. Adriani et al. (PAMELA Collaboration), Science 332 (2011) 69

[4] A.D. Panov et al. (ATIC Collaboration), Bull. Russ. Acad. Sci.: Phys. 73 (2009) 564

[5] J. Chang et al. (DAMPE Collaboration), Astropart. Phys. 95 (2017) 6-24 